\title{
Abundances in Magellanic Cloud Young Populous Clusters
}

\author{
V. Hill ${ }^{1}$ \\ ESO, K. Schwarzschild Str. 2, D-85748 Garching b. München, \\ Germany
}

\begin{abstract}
Detailed abundances are measured in cool supergiants in Magellanic Cloud young populous clusters (NGC 330 in the SMC, NGC 2004, NGC 2100 and NGC 1818 in the LMC) and compared to those recently observed in samples of field supergiants in each Cloud. The analysis of the field supergiants shows no evidence of any abundance dispersion among the stars, compatible with a well mixed gas in these galaxies. However, previous determinations of the metallicity of NGC 330 and NGC 1818 give values well below the respective field values. This difference is investigated using a homogeneous method for the field and cluster samples, and the abundance ratios of various elements are examined.
\end{abstract}

\section{Introduction}

The Magellanic Clouds' young stellar population includes a number of so-called populous clusters, which have no counterpart in our own galaxy. Very young $\left(10^{6}\right.$ to $\left.10^{7} \mathrm{yrs}\right)$ and yet very rich, these clusters have long been targets both for cluster formation and stellar evolution studies. Among these clusters, particular attention was drawn to NGC 330 (SMC) and NGC 1818 (LMC), since early attempts to measure their metallicities indicated a low metal content (lower than that of the surrounding stars of similar ages, by factors of 5 and 4 respectively: Carney et al. 1985; Richtler et al. 1989). How could a cluster form with a metallicity lower than that of its surroundings? This is especially peculiar since the young population in the field of the MCs seems to be fairly well mixed: no abundance gradients or large scatter are found among the supergiants (Hill et al. 1995; Hill 1997; Luck et al. 1998), nor among the H II regions (Russell \& Dopita 1990). Further spectroscopic studies confirmed that NGC 330 was indeed more metal poor than its surroundings (Spite et al. 1991). However, the reddening and hence the metallicity for this cluster has been questioned (Bessell 1991). The real metal deficiency of the MC young clusters is thus still not understood.

Our recent study of six K-type supergiants in the SMC field (Hill 1997) provides an ideal reference sample for this type of star. Taking advantage of this, we undertook a study of similar stars in four MC Young Populous Clusters. With the same models, methods and physical data being used for all stars, most of the systematic uncertainties cancel out when differential abundances (clusters

${ }^{1}$ IAG, Universidade de São Paulo, CP 3386, São Paulo 01060-970, Brazil 


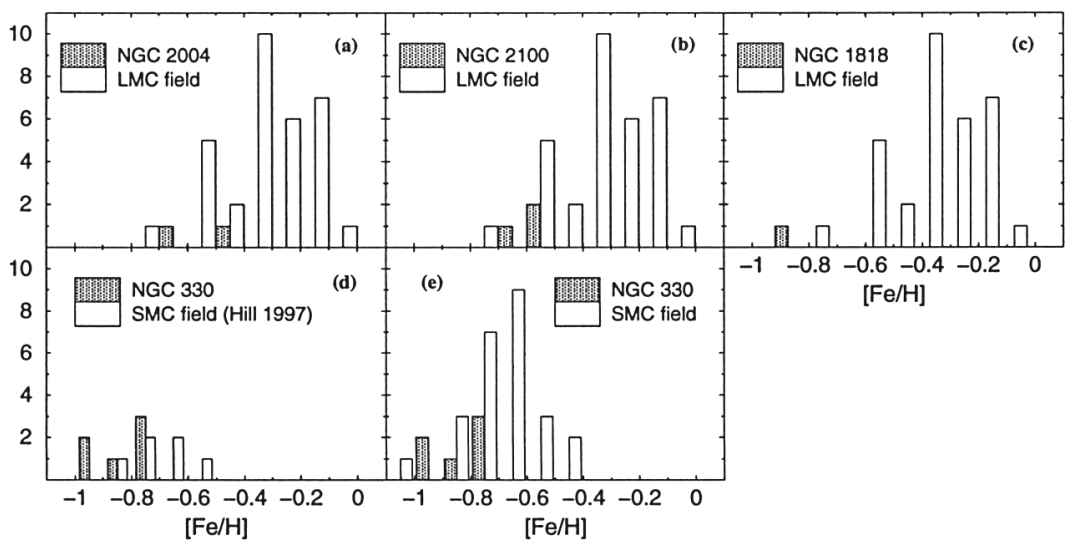

Figure 1. Histograms of the $[\mathrm{Fe} / \mathrm{H}]$ distribution of cluster and field stars in the LMC (top panel) and SMC (bottom panel). (a) NGC 2004 (this work) and the LMC field (compilation by Luck et al. 1998.(b) the same for NGC 2100. (c) the same for NGC 1818.(d) NGC 330 and the SMC field (Hill 1997). (e) NGC 330 and the SMC field (Luck et al. 1998).

versus field) are considered. The program was conducted at the NTT (ESO) where spectra were obtained for a sample of cool supergiants in four MC young clusters: NGC $330(\sim 20 \mathrm{Myrs})$ in the SMC, NGC 1818 ( 17 Myrs), NGC 2004 ( $\sim 8 \mathrm{Myrs})$ in the LMC, and NGC $2100(\sim 12 \mathrm{Myrs})$ in the 30 Dor region of the LMC. The results for six stars in NGC 330 (SMC) were reported in Hill (1997b) and show that the difference, if any, between the cluster and the field stars is small and compatible with the small scatter found among the field stars. The LMC clusters are still under analysis, so the results reported here come from roughly half of the available data.

\section{Cluster Versus Field Stars}

The mean iron abundances found for the LMC clusters from the program stars are respectively: $[\mathrm{Fe} / \mathrm{H}]=-0.91(\mathrm{NGC} 1818,1 \mathrm{star}),[\mathrm{Fe} / \mathrm{H}]=-0.57(\mathrm{NGC} \mathrm{2004}$, 2 stars) and $[\mathrm{Fe} / \mathrm{H}]=-0.57 \pm 0.06\left(\sigma_{r m s}\right)$ (NGC 2100, 3 stars).

The histograms in Fig.1 show that only in NGC 1818 are the field and cluster stars strikingly different. There still seems to be a systematical tendency of the four clusters (the three LMC clusters and NGC 330 in the SMC) to be more metal deficient than their surroundings, but the difference is small $(<0.2$ dex) and hardly significant in all cases except NGC 1818 . However, since only one star in NGC 1818 has been analyzed so far, no firm conclusion can be drawn about this apparently peculiar cluster until other stars are analyzed.

Panels (a) and (b) of Fig.2 display the mean [X/Fe] ratios versus atomic number for the field (Hill 1997; Hill et al. 1995) and the LMC and SMC cluster (this work) samples. Panel (c) shows the difference between the mean for each cluster and the mean of the SMC K-type supergiants sample $\left([\mathrm{X} / \mathrm{Fe}]_{\text {cluster }}\right.$ 
- $\left.[\mathrm{X} / \mathrm{Fe}]_{\text {fieldSMC }}\right)$. This plot shows that the difference in the detailed chemical composition of the clusters and the field stars of the MCs is extremely small, seldomly exceeding the intrinsic expected uncertainty (dotted lines). The striking similarity of the abundance patterns favors a formation of young clusters from matter which evolved similarly to that of the bulk of the MCs.

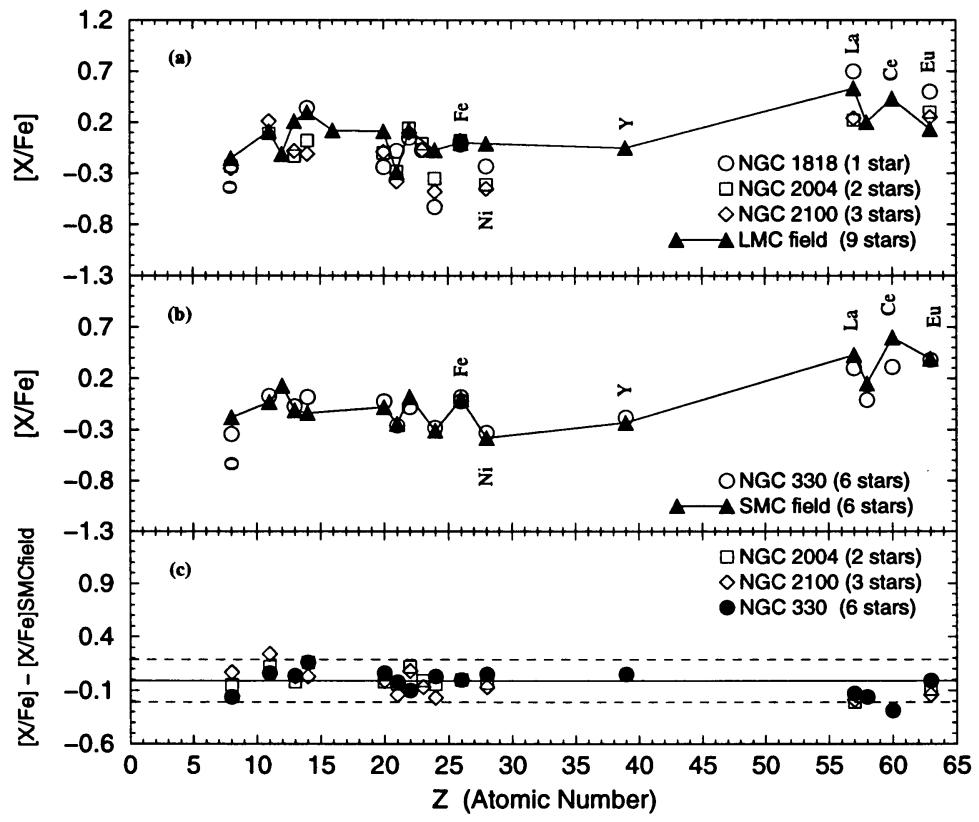

Figure 2. (a) mean abundances relative to iron of the LMC clusters and the field, plotted as a function of atomic number. (b) same as (a) for NGC330 and the surrounding SMC field. (c) Residual [X/Fe] abundances of the clusters when normalized to the SMC field $\mathrm{K}$ stars.

\section{3. $\quad \alpha$ Elements and Heavy Neutron Capture Elements}

Oxygen and $\alpha$ elements are of particular interest for the study of the chemical evolution of a galaxy because they are thought to be produced in large quantities by the short-lived type II supernovae (SNIIe). This is in contrast to iron which is only marginally produced by SNIIe and more efficiently produced in the longer lived type Ia supernoave. It is now widely accepted that the $\alpha$ elements are not overabundant in the young matter of the Magellanic Clouds (in contrast with the Galactic dwarfs of similar metallicity). This can clearly be seen in Fig.3 (left), where both the field and cluster $\mathrm{K}$ supergiant $[\mathrm{O} / \mathrm{Fe}]$ values are plotted for both MCs, together with Galactic data (the dashed box represents the location of the solar neighbourhood supergiants). Also striking is the similarity of the Magellanic and Galactic supergiant oxygen to iron ratios. This feature may be simply explained by a star formation history which occurs more slowly in the Clouds than in the Galaxy; a bursting star formation history could also produce 

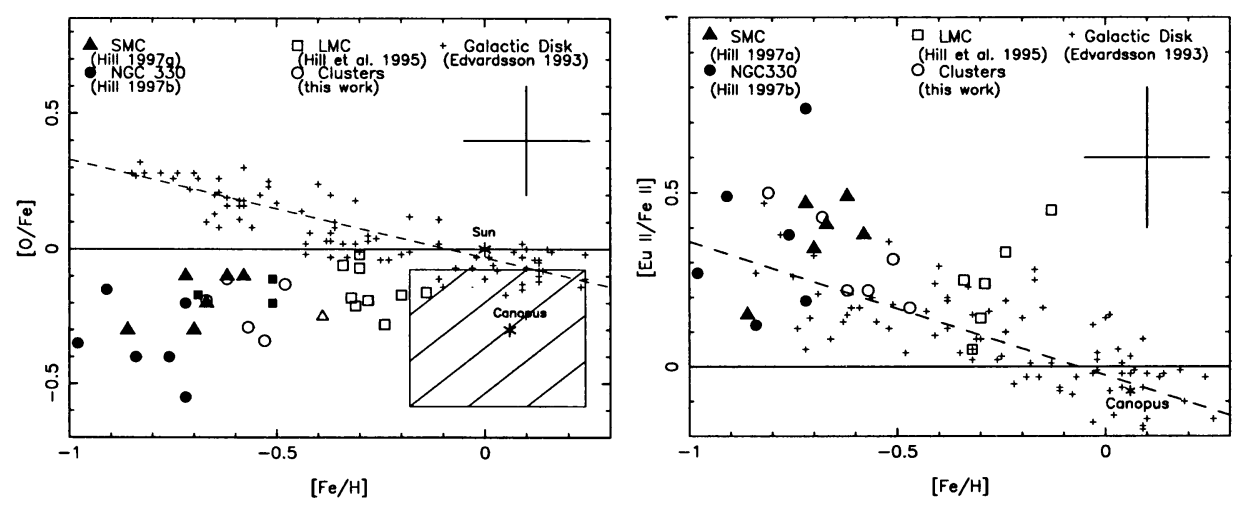

Figure 3. $\quad[\mathrm{O} / \mathrm{Fe}]$ and $[\mathrm{Eu} / \mathrm{Fe}]$ ratios as a function of $[\mathrm{Fe} / \mathrm{H}]$ for supergiants in both clouds (field and clusters) and in the Galaxy.

such $[\mathrm{O} / \mathrm{Fe}]$ ratios in the young population. In either cases there is no need for a IMF different in the Clouds and the Solar neighbourhood.

On the other hand, the heavy $r$-process neutron capture elements (Europium is the best example) are also thought to be produced mainly by SNIIe. In the disk of our Galaxy, the behaviour of $[\mathrm{Eu} / \mathrm{Fe}]$ indeed resembles that of the $[\mathrm{O} / \mathrm{Fe}]$ (Fig.3). However, in the MCs, the heavier $s$ - and $r$-process neutron capture elements are found to be significantly overabundant (by $\sim 0.3$ and $\sim 0.4$ dex respectively both in field and cluster stars). In the right panel of Fig.3 the $[\mathrm{Eu} / \mathrm{Fe}]$ values for field and clusters in both MCs are plotted. The small symbols are the data for the Galactic disk (Woolf et al. 1995).

This difference of behaviour between $\alpha$ and $r$-process elements in the MCs is very difficult to understand, since both types of elements are thought to be produced in SNIIe and more work deserves to be done on this topic to unravel the true history of chemical enrichment in the Clouds.

Acknowledgments. This research was partly supported by Fapesp.

\section{References}

Bessell, M. 1991, in IAU Symp. 147, Haynes R., Milne D. (eds.), Kluwer, p.273

Carney, B.W., Janes, K.A., \& Flowers, P.J. 1985, AJ, 90, 1196

Hill, V. 1997, A\&A, 324, 435

Hill, V., Andrievsky, S., \& Spite, M. 1995, A\&A, 293, 347

Luck, E., Moffett, T., Barnes, T., \& Gieren, W. 1998, ApJ, 115, 605

Richtler, T., Spite, M., \& Spite, F. 1989, A\&A, 225351

Russell, S., \& Dopita, M. 1990, ApJS, 74, 93

Spite F., Spite M., \& Richtler, T. 1991, A\&A, 252, 557

Woolf, V., Tomkin, J., \& Lambert, D.L. 1995, ApJ, 453, 660 


\section{Discussion}

Jay Gallagher: Can you say anything about the dispersion in abundance among the young stars in the LMC from your data?

Hill: From our $9 \mathrm{~F}$ supergiants in the LMC, we could see no real dispersion of abundances $(\sigma=0.07 \mathrm{dex})$. To my understanding, the scatter that remains in the total distribution of $[\mathrm{Fe} / \mathrm{H}]$ values is due to differences in the various authors' approaches.

Dominik Bomans: You stressed the importance of differential analysis. Microturbulence is critical. How similar are the field and cluster samples in terms of microturbulence and how does it affect your results?

Hill: Both samples in SMC field and cluster display the same range of microturbulence velocity $\left(3-3.5 \mathrm{~km} \mathrm{~s}^{-1}\right)$. All the more, given the range of lines of various intensities that are observed, the microturbulence velocities are derived in a rather reliable way.

Johannes Anderson: The picture of a dispersion of $0.2-0.3$ dex in $[\mathrm{Fe} / \mathrm{H}]$ and other ratios $[\mathrm{X} / \mathrm{Fe}]$ is, of course, just what is seen in the MW disk, except for $[\mathrm{O} / \mathrm{Fe}]$. Which lines were used to determine the $[\mathrm{O} / \mathrm{H}]$ ?

Hill: The oxygen abundance is derived from the forbidden [O I] $6300 \AA$ line. 